\title{
Concepto y objeto. La caja de artista como recurso interdisciplinar en educación primaria
}

\section{Concept and object. The artist box as an interdisciplinary resource in primary education}

\author{
Encarna Monteagudo ${ }^{1}$ \\ encarna@ucv.es \\ Eva Muñoz Guinea \\ eva.munoz@ucv.es \\ Universidad Católica de Valencia San Vicente Mártir, España
}

\section{Resumen:}

El presente artículo plantea "La Caja" como objeto artístico conceptualizado como material didáctico. Tomando como antecedente la Boîte-en-Valise de Marcel Duchamp y las bases del arte conceptual, la caja como objeto artístico plantea, dentro de los procesos de enseñanza-aprendizaje, una herramienta activa que puede llegar a ser contenedora de conceptos. De este modo, el proyecto "Caja de artista" pretende procesar aspectos curriculares desde una óptica abierta, creativa y que promueva la consecución de competencias básicas.

Tras un acercamiento a la aparición y desarrollo de la caja como elemento artístico a lo largo del siglo XX, se exponen los resultados de un estudio realizado con estudiantes de Grado en Maestro de Educación Primaria de una Facultad de Magisterio y Ciencias de la Educación de la Comunidad Valenciana. En base a los proyectos realizados en el área de Educación

\begin{abstract}
:
This article presents "The Box» as an artistic object conceptualized as a didactic material. Based on the Boitte-en-Valise by Marcel Duchamp and the foundations of conceptual art, the box as an artistic object is conceived, within the teaching-learning processes, as an active tool that can become a container of concepts. In this way, the "Artist Box» project aims to process curricular aspects from an open, creative perspective that promotes the achievement of basic skills. After studying the emergence and development of the box as an artistic element throughout the twentieth century, the results of a study carried out with students of the Master's Degree in Primary Education at a Valencian University will be reported. Drawing on the projects carried out in the area of Artistic Education, the creation process occurred is described together with an the evaluation which students provided by completing questionnaires along the implementation
\end{abstract}

1 Dirección para correspondencia (correspondence address):

Encarna Monteagudo García. Departamento de Enseñanza-aprendizaje de la educación física, plástica y musical. Facultad de Magisterio y Ciencias de la educación. Universidad Católica de Valencia San Vicente Mártir. C/ Sagrado Corazón, n5. 46110, Godella, Valencia (España). 
Concepto y objeto. La caja de artista como recurso interdisciplinar en educación primaria

ENCARNA MONTEAGUDO Y EVA MUÑOZ GUINEA

Artística se describe el proceso de creación así como una evaluación sobre diferentes aspectos en el desarrollo del mismo en base a cuestionarios cumplimentados por los estudiantes.

Así mismo, se exponen cuestiones referentes a una puesta en práctica llevada a cabo por estudiantes de Grado en aulas de educación primaria de un CEIP de la Comunidad Valenciana. Para finalizar, se analiza un cuestionario lanzado a los profesores del centro educativo implicados con el fin de extraer una serie de consideraciones sobre esta propuesta artística como posible material didáctico.

\section{Palabras clave:}

Caja de artista; arte conceptual en educación; proyecto interdisciplinar. of the experience. The present study also reports on some issues emerging from the implementation of the same experiment by Primary Education Degree students with Primary School students at a Valencian school. Finally, a questionnaire sent to the teachers of that school are analyzed in order to extract a series of considerations on this artistic proposal as a possible didactic material.

\section{Key words:}

Artist box; conceptual art in education; interdisciplinary project.

\section{Résumé:}

Cet article présente «The Box» comme un objet artistique conceptualisé comme matériel pédagogique. En se basant sur la Boîte-en-Valise de Marcel Duchamp et sur les bases de l'art conceptuel, la boîte comme objet d'art est conçue, dans le processus d'enseignement-apprentissage, comme un outil actif qui peut être conteneur de concepts. Ainsi, le projet «artiste Box» vise à traiter les aspects scolaires dans une perspective ouverte, créative et favorise le développement des compétences de base.

Après l'apparition et le développement de la boîte comme élément artistique tout au long du XXe siècle, les résultats d'une étude des étudiants de Grado en enseignement primaire de la Faculté d'Education et en Sciences de l'Education sont exposés à Valencia. À partir des projets réalisés dans le domaine de l'éducation artistique nous décrivons le processus de création, ainsi qu'une évaluation des différents aspects de leur développement à partir de questionnaires remplis par les étudiants décrits.

En outre, des questions concernant nous exposons des questions liées à une mise en œuvre réalisée par les étudiants de premier cycle dans les classes des écoles primaires de Valencia. Enfin, nous analysons un questionnaire envoyé aux enseignants de l'école impliquée afin d'en tirer un certain nombre de considérations sur cette proposition artistique en tant que matériel pédagogique possible.

\section{Mots clés:}

Boîte Artiste; l'éducation artistique conceptuelle; projet interdisciplinaire.

Fecha de recepción: 23-11-2016

Fecha de aceptación: 20-5-2017 


\section{Introducción}

Los propios principios educativos y competenciales marcan la importancia de estrategias y medios que diversifiquen el aprendizaje, sean motivadores y conduzcan a la acción y la autonomía de los estudiantes. La educación artística puede dar respuesta a retos tan importantes como el desarrollo de la creatividad, la motivación al aprendizaje de otras disciplinas así como la adquisición de competencias. Tal y como plantea la Hoja de ruta para la educación artística existen dos enfoques principales de la educación artística que pueden implementarse al mismo tiempo y no necesariamente por separado; En concreto, las artes pueden ser disciplinas artísticas que tengan como fin sensibilizar y apreciar las artes y desarrollar competencias propias y, por otro lado, "utilizar como método de enseñanza y aprendizaje e incluir dimensiones artísticas y culturales en todas las asignaturas del currículo" (UNESCO, 2006, p.5).

Para Arnheim (1993) el lugar de la educación artística debería funcionar como una de las áreas de aprendizaje cuya misión fuera "dotar a la mente del joven de las habilidades básicas para afrontar con éxito todas las ramas del currículum" (p.89). En este sentido, el arte es un medio para el desarrollo cognitivo y puede ser entendido como el eje que sustenta otros aprendizajes. De este modo, el desarrollo de capacidades y conocimientos a través de la creación artística, partiendo de los propios intereses de los discentes, se pone de manifiesto en la propuesta expuesta en este artículo. Dicho proyecto pretende dinamizar procesos de enseñanza-aprendizaje de cualquier área del currículo escolar desde una óptica artística; este planteamiento es respaldado por la UNESCO (2006): "hay que animar a los profesores de asignaturas artísticas a aprovechar las competencias de otros artistas, incluso de otras disciplinas, y, al mismo tiempo, adquirir las competencias necesarias para colaborar con artistas y con profesores de otras asignaturas en un contexto educativo" (p.7).

Tomando como punto de partida las premisas expuestas, se explica un proyecto artístico Ilamado "Caja de artista" ${ }^{2}$ "levado a cabo en una Facultad de Magisterio y Ciencias de la Educación de la Comunidad

2 El proyecto ha sido Ilamado "Caja de artista" debido a las coincidencias conceptuales y artísticas que se encontraron con el "Libro de artista" denominación utilizada para libros nacidos en el marco del arte conceptual. Como indica Vásquez (2013), en las cajas-maletas y libros de artista el concepto está relacionado con la investigación sobre el lenguaje en un soporte artístico. 
Valenciana. La experiencia se ha planteado, en el área de Educación Artística a un total de 120 estudiantes del Grado en Maestro de Educación Primaria de tercer curso. Dicha propuesta versa sobre un elemento aparentemente sencillo como es una caja. Partiendo de las bases del arte conceptual, y huyendo de formatos más tradicionales, tales como la pintura o la escultura, el estudiante puede bajo estos parámetros, abordar cualquier técnica artístico-plástica para conformar, en lo que se refiere a contenido y continente, un objeto. Desde una óptica abierta, los estudiantes configuran un discurso en el cual, tal y como el arte conceptual plantea, la idea o concepto prevalece sobre el objeto artístico.

Se plantea, por lo tanto, un proyecto educativo que convierte en protagonista de sus propios procesos de enseñanza-aprendizaje al estudiante; el proyecto "Caja de artista" pretende organizar el pensamiento, manipular contenidos, procesar información, crear materiales didácticos propios y desarrollar la competencia, sobre otras, de aprender a aprender mediante la interdisciplinaridad, tal y como plantea la Hoja de ruta para la educación artística:

el enfoque de las artes en la educación pretende poner los beneficios de la educación artística al alcance de todos los alumnos en todas las asignaturas. Este enfoque también tiene como objetivo contextualizar la teoría a través de la aplicación práctica de disciplinas artísticas. Para que resulte eficaz, este enfoque interdisciplinario exige modificar los métodos y la formación del profesorado (UNESCO, 2006, p.6).

\section{Marco teórico}

\section{La caja como concepto artístico}

La caja como obra artística puede ser entendida como concepto, partiendo de la idea de "objeto" al margen de formatos pertenecientes a la estructura expositiva, teniendo, como antecedente más evidente las "Boîte-en-Valise" de Marcel Duchamp de 1914. En ella, Duchamp conceptualizó este espacio cerrado, el propio artista dijo sobre esta obra:

Otra nueva forma de expresión. En lugar de pintar algo, se trataba de reproducir esos cuadros que tanto me gustaban en miniatura y a un volumen 
muy reducido. No sabía cómo hacerlo. Pensé en un libro, pero no me gustaba la idea. Entonces se me ocurrió la idea de la caja en donde todas mis obras se hallarían recogidas como en un museo en miniatura, un museo portátil, y eso explica que lo instalara en una maleta (Duchamp, 1978, p.161).

La Boîte Verte, Blanche y la Boite de 1914, tienen un sentido abierto documental. En ellas Duchamp completa conceptualmente sus obras -como El gran vidrio- o almacenan ideas, reflexiones o metaironías.

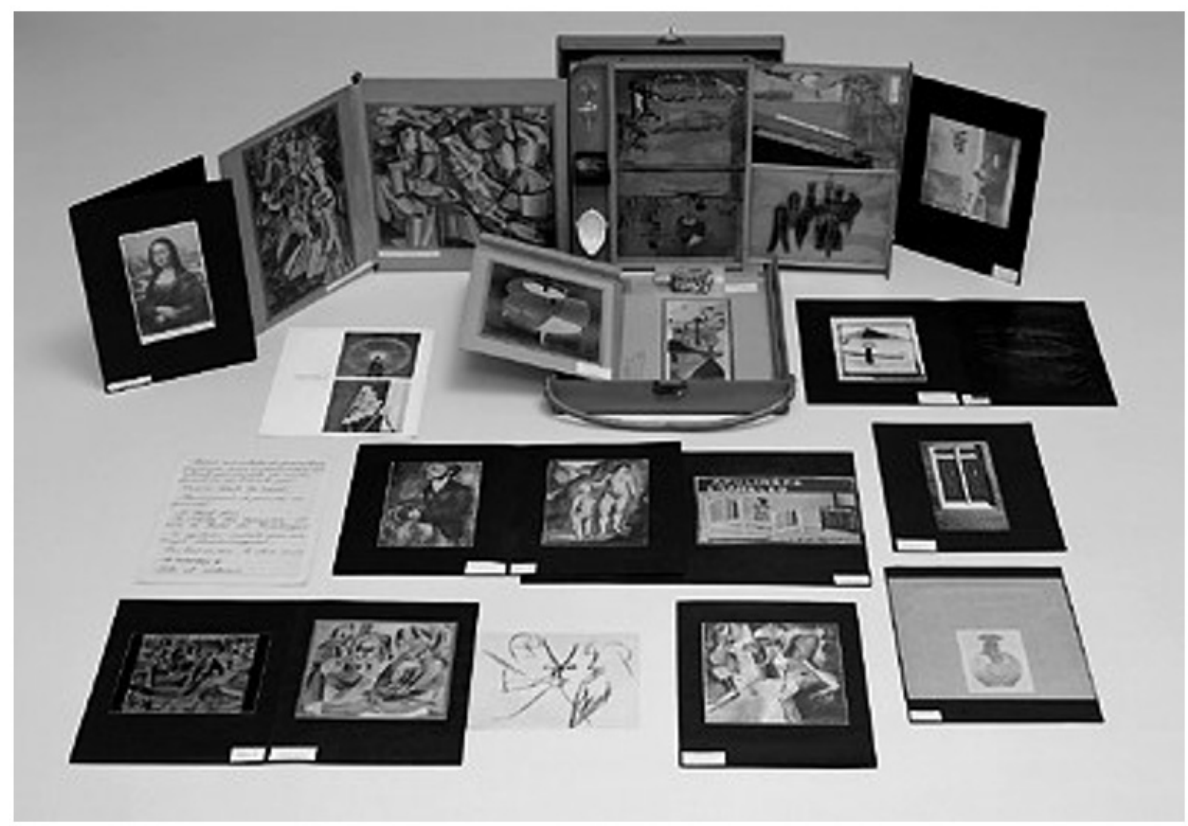

Figura 1. Boîte-en-Valise (1935-41) de Marcel Duchamp. Recuperado de https://www.moma.org/interactives/exhibitions/1999/muse/artist_pages/duchamp_boite.html,

El pintor mejicano Vicente Rojo revisita el concepto planteado por Duchamp, partiendo del texto de Octavio Paz dedicado al artista, para complementarlo visualmente: "es un libro fuera de concepciones. La caja de Duchamp la puedes ver, tocar. Yo quería recordar la figura de Duchamp, marcar su importancia" (Rojo como se citó en González, 2014, p.247). Pero más que un libro visual o de artista, el libro-maleta se trata de "un archivo, una biblioteca, caja, museo portátil" (González, 2014, p.247). 
En un sentido más evocador, Joseph Cornell desarrolló un mundo poético dentro de cajas, construidas en madera o vidrio que "contienen una máquina de símbolos, conjunción de idea, inspiración y tiempo suspendido" (López, 2006, p.330). Pequeños espacios oníricos, algunos llamados "Museo", donde se mezclan estética surrealista con recuerdos de anticuario. Para Foster, Krauss, Bois y Buchloh (2001) se trata de "juguetes filosóficos que crean una estética de prodigio, espacios oníricos donde arena y estrella, o pompas de jabón y mapas lunares, parecen tocarse, deja que la subjetividad sea coherente a través del medio de la memoria" (p. 254).

En Box with the Sound od Its Own Making (Caja con el sonido de su propia producción de 1961), Morris realiza un objeto escultórico en forma de caja, en una primera lectura podría tratarse de un cubo pero el artista plantea un contenido inmaterial: se trata de sonidos grabados durante el proceso de producción del objeto artístico, "sonidos que emergen desde su interior, desvelan que esta forma aparentemente $<<$ pura $>>$ es en realidad un híbrido de la memoria, de la textura, del sonido y de la tecnología de producción que han dado lugar a este objeto, supuestamente dueño de sí mismo" (Foster, et al., 2001, p. 529). Dentro de un conceptualismo similar, Card File, del mismo autor, es un archivador de tarjetas con notas sobre la producción del propio objeto, "consiste en el registro escrito de todos los encuentros azarosos que formaron parte del proceso de producción (...) su aleatoriedad incluso en su trivialidad (vinculado a la estética Fluxus y pop), no son extrínsecos al objeto estético, sino que se vinculan a él" (Foster, et al., 2001, p.529). El conceptualismo de las dos obras, unido a su contenido y sentido, viene marcado por la idea de proceso al margen de la obra. Caja y archivador, aunque pudiera parecer relevante como escultura, funcionan como contenedor de "datos procesuales".

Parece evidente, por lo tanto, que el interés primordial del movimiento fluxus no eran los objetos artísticos sino la evocación de lo efímero, lo trivial y el proceso o acción. Este movimiento tiene un objeto "típico", aparentemente poco espectacular, pequeño y empaquetado: la idea de caja (derivada del estuche en maleta de Duchamp). A partir de 1962 es desarrollada por Maciunas produciendo ediciones de maletas de piel. Las Flux-kist albergan un conjunto de objetos y artículos impresos por artistas que orbitan alrededor de Maciunas los cuales habían contribuido en festivales y eventos organizados por el grupo desde 1962. Los male- 
tines podían adquirirse a un módico precio, fue uno de los productos más elaborados de la Ediciones Fluxus, Ilenos de pequeños objetos para leer y manipular. Los contenidos varían entre cada kit pero solían incluir puzzles, collages, múltiples, cartas sugerentes o discos. Entre los contribuidores más asiduos se encontraban Brecht, Robert Wats, Ay.O, Joe Jones, Oldenburg y otros artistas que aportaron objetos (MOMA, 2011).

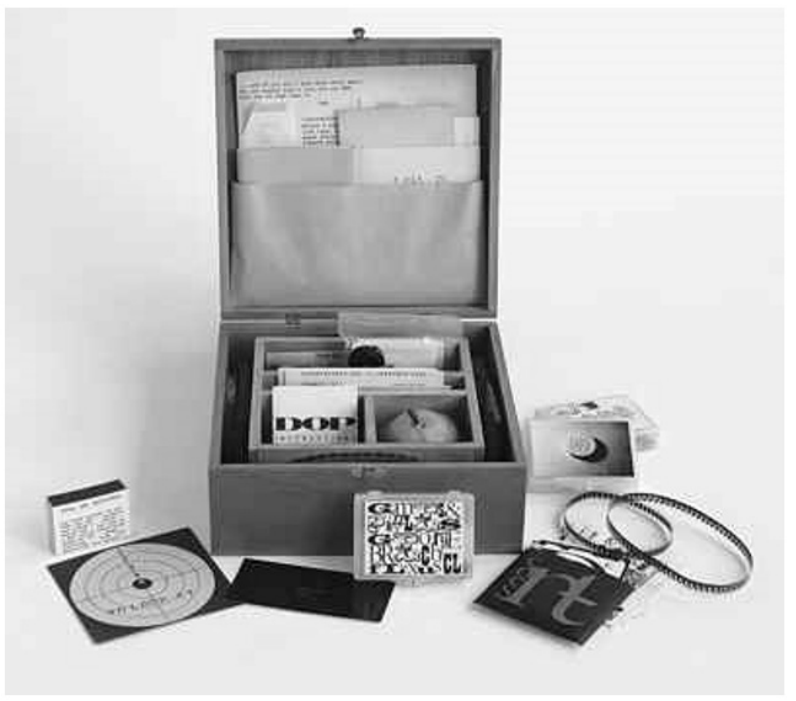

Figura 2. Flux Year Box 2. Maciunas1968, MOMA (2011). Recuperado de https://www.moma.org/interactives/exhibitions/2011/fluxus_editions/works/ year-box-2/index.html

De este modo, la caja como concepto, vitrina expositiva, caja de sonidos o archivador de procesos, trasladan el objeto de arte al terreno de la idea. Duchamp abrió una brecha difícil de cerrar, con el ready-made y sus Boîte-en-Valise, estableció las bases del arte como concepto, creando el campo de cultivo de gran parte de las manifestaciones artísticas del siglo XX y XXI.

\section{La caja como concepto didáctico}

Una caja puede ser un lugar donde almacenar, donde poder esconder las peculiaridades de cada uno, los recuerdos, los tesoros, los conocimientos. Dentro de un aula este pequeño espacio está esperando a ser 
Ilenado; metafóricamente, el aula puede moverse mediante una caja que puede transportarse $y$, de este modo, no es necesario estar entre cuatro paredes. A la hora de desarrollar determinados procesos de aprendizaje, el "abandono temporal del aula" (con una caja como material de apoyo didáctico), se convierte en un recurso donde archivar y evocar. De este modo, una caja puede llevarse al lugar de investigación, allí donde se encuentre la fuente de conocimiento. Un cuaderno de viaje, una cámara de fotos o un Ipad, puede recoger recuerdos (lo que se ha dicho, leído, escuchado o hecho), pero una caja puede contener todo esto, juntarlo con un propósito, procesarlo y, además, dotarlo de entidad artística.

Así mismo, una caja que alberga tesoros encontrados puede ser un "escondite" con el que proteger los hallazgos, los conocimientos guardados en la intimidad de un lugar que sólo se abrirá cuando su dueño/s lo deseen. A propósito de esto, Tonucci dice sobre la utilidad de la caja:

La caja se transforma...en una caja, en un cuadro profundo, donde se puede $<<$ pintar $>>$ con cosas $<<$ bonitas $>>$ que se encuentran por ahí, cambiando de nuevo su sentido originario, disponiéndolo $<<$ bien $>>$.. De pequeño hice un agujero en la tierra, metí dentro bolitas de papel de plata de varios colores, pedacitos de vidrio pintado (desgastados por el mar), y lo cubrí con un cristal: era mi tesoro. La caja se convierte en un cofre de un tesoro hecho de plumillas, de llaves, de puntillas, de dibujitos, de cajas más pequeñas que contiene cosas más pequeñas aún. (Tonucci, 2005, pp.75, 76).

Por otro lado, además de su contenido, también puede considerarse el mismo espacio interior de la caja como elemento conceptual, Ilegando a ser su máxima expresión precisamente, debido a la ausencia de cualquier elemento en el mismo. A este respecto, Bosch (2009) cuenta sus impresiones cuando conoció la exposición sobre Jorge Oteiza celebrada en Barcelona "La Caja vacía", y reflexiona sobre sus evocaciones ante la aseveración del escultor al decir "¡con nada!":

¡Con nada! Es un compendio educativo completo. Lo aprendí también en la contemplación de la Caja vacía. ¿Qué otra cosa se puede hacer en educación si no se comienza por crear consciencia del vacío hasta ser capaces de reconocer el espacio disponible? No es precisamente esta disponibilidad el punto de anclaje de la singularidad de cada uno? ¿No era este proceso de reconocimiento propio del que hablaban las cajas de cartón transformadas 
en cajas mágicas? Y si es así, por qué las escuelas no disponen aún de lugares vacíos en los cuales almacenar el futuro de los escolares que comienzan? (Bosch, 2009, p.171).

Así pues, la "Caja de artista" es un "lugar" dónde guardar lo que se desee y necesite recordar; en primer lugar, el acto de guardar, reorganizar y construir el propio aprendizaje puede entenderse, por sí mismo, como un acto inherentemente creativo. En segundo lugar, como espacio vacío en el que se sistematizará los materiales didácticos y que puede ser entendido como concepto artístico. De este modo, se plantea un intento estético-conceptual de elevar a categoría artística la documentación que puede albergar una caja. Contenido y continente por lo tanto, pretende ser evocación y homenaje al conceptualismo de Marcel Duchamp, al onirismo de Josep Cornell, a lo cotidiano y casual de Fluxus y sobre todo, al acto creativo que ha sido siempre "encajonar" hallazgos y "tesoros" llevado a cabo por los niños.

\section{Marco empírico}

Expuestos ejemplos y sus bases estético-conceptuales, se plantea la viabilidad que este objeto artístico puede suscitar como proyecto artístico para estudiantes de grado de magisterio, así como para alumnos que cursan educación primaria. En este sentido se pretende:

- Exponer los planteamientos sobre el proyecto "Caja de artista" en el área de Educación Artística a estudiantes de Grado en Magisterio de Educación Primaria de una Facultad de Magisterio y Ciencias de la Educación de la Comunidad Valenciana.

- Valorar diferentes aspectos sobre el proceso de creación de esta obra conceptual, así como apreciaciones sobre logros académicos y posibilidades profesionales por parte de 120 estudiantes que desarrollaron el proyecto.

- Comprobar la aceptación de alumnos y alumnas de $1^{\circ}$ a $6^{\circ}$ de primaria del centro educativo y establecer el grado de satisfacción sobre diferentes aspectos de "La caja de artista" desde una dimensión didáctica por parte de los 12 profesores del centro implicados en la puesta en práctica. 


\section{Metodología}

En base a los planteamientos conceptuales, se muestran, por un lado, las fases de creación de dicho proyecto y un ejemplo de las partes constituyentes de la obra de una estudiante. Por otro lado, se analizan cuestionarios finales cumplimentados por 120 alumnos en los que responden a cuestiones sobre el desarrollo de su obra en diferentes aspectos académicos y posibilidades profesionales que, como material didáctico pudiera tener.

Posteriormente se realiza una puesta en práctica en un CEIP, con alumnos de $1^{\circ}$ a $6^{\circ}$ de primaria. Con el fin de comprobar la idoneidad de esta propuesta en dicha etapa, se analizan 12 cuestionarios lanzados a los profesores-tutores de aula que presenciaron la interactuación de sus alumnos primaria con los estudiantes de grado en magisterio. Las preguntas versaban sobre el propio objeto artístico y sobre la aceptación de los discentes, así como, sobre las posibilidades que dicho material podría suscitar en los procesos de enseñanza-aprendizaje.

\section{Desarrollo del proyecto "Caja de artista"}

El binomio caja de arte conceptual y contenedor de contenidos curriculares es la base, por lo tanto, de la propuesta artística para los estudiantes que aquí se presenta. Para ello, se tiene en cuenta que, el contexto cultural del que se nutre la educación artística y por extensión la nueva concepción del arte, marcan el relativismo estético y el valor del objeto artístico. En base a esto, el proyecto "Caja de artista" se plantea a los estudiantes como un objeto de arte conceptual. Dicha caja debe albergar materiales didácticos de elaboración propia, juegos o juguetes manufacturados, documentación gráfica, libro de artista o soportes audiovisuales realizados por el autor/a. Todo ello tenía que poseer un carácter coherente de conjunto en cuanto a códigos artísticos se refiere. Por otro lado, el estudiante debía utilizar al menos cuatro técnicas plásticas y/o visuales bidimensionales y una tridimensional. Estas fueron las únicas consignas que se proporcionaron y que pretendían invitar a la investigación conceptual, técnica y procedimental para el diseño y desarrollo del proyecto.

En la primera toma de contacto con los estudiantes en el aula, se plantearon las principales características del arte conceptual mediante el visionado y análisis de diferentes ejemplos, para establecer un debate 
posterior sobre las bases de esta corriente artística así como sobre su contextualización. Con ello se pretendió que los estudiantes plantearan sus dudas sobre la obra artística "sin valor técnico", dentro de un contexto artístico en donde el proceso y la idea priman sobre el resultado final. No obstante, una vez planteada la caja como idea y excusa para la creación artística, se delimitó su sentido y utilidad didáctica. Para ello, en un segundo momento se planteó que este proyecto artístico debía responder a resultados de aprendizaje tales como la utilización de elementos propios de la sintaxis visual y la utilización de técnicas y recursos propios de la expresión plástica y visual. En este punto hubieron de plantearse las fases del proceso en base a un determinado abanico de posibilidades: utilización de técnicas bidimensionales (collage, pintura, fotografía), tridimensionales (volúmenes, modelados, vaciados) así como la utilización de cualquier tipo de material audiovisual.

Añadido a la importancia del diseño y de la planificación de la propuesta, se planteó además la cuestión de que este objeto conceptual debía ser un material didáctico para el aula de primaria, de manera que la excusa para la creación, debía partir de contenidos del currículo escolar (Decret 108/2014 de 8 de julio, del Consell, por el que se establece el currículo y desarrolla la ordenación general de educación primaria en la Comunidad Valenciana). Se confió, en cualquier caso, en la autonomía en cuanto al procesamiento de información por parte del estudiante, de modo que, tras la elección del tema, éste buscase las soluciones estéticas y técnicas adecuadas para abordar el proyecto. De esta manera, se tiene en cuanta las investigaciones llevadas a cabo por Freedman, que han mostrado que cuando los estudiantes se enfrentan "a una nueva forma visual, el foco de la cognición suele ser más la relación entre la forma visual y sus significados asociados, que el objeto particular" (Freedman como se citó en Aguirre, 2005, p.315). Según Aguirre (2005):

los estudiantes ya están culturalmente orientados hacia la $<<$ intergraficalidad $>>$, es decir a la conexión entre códigos gráficos de distinta naturaleza estética, y según Freedman, ésta es una habilidad que debe ser tenida en cuenta en elaboración y desarrollo de un currículum postmoderno de arte" (p.315).

Una vez elegido el contenido o competencias que cada estudiante deseó desarrollar, se le acompañó en el aula-taller para que planificase 
las fases de su proyecto, considerando que la labor del profesor, "es ayudar a los alumnos a explorar las representaciones desde una perspectiva interdisciplinaria y considerar la representación visual como una cuestión de convenciones" (Bryson y Holly como se citaron en Aguirre, 2005, p.315).

La segunda elección fue encontrar o realizar la caja que contendría la información desglosada, instalada o planteada en su interior. De este modo, el estudiante delimitó qué aspectos deseaba tratar y bajo qué parámetros artísticos, recordando que él era el principal agente implicado en su aprendizaje, de manera que el tema, las técnicas y los conceptos a tratar fueran adecuados a sus intereses, capacidades y preferencias. En este sentido, se persigue un fortalecimiento personal de las capacidades formativas del alumno a través de la variedad de posibilidades técnicas y temáticas de las que dispone: "la diversificación de los recursos es el alimento para el desarrollo plural de las inteligencias" (Eisner como se citó en Aguirre, 2006, p.94). El estudiante eligió la vía, el método, las técnicas y los temas que mejor se adecuaron a sus intereses y actitudes. El proceso, elaboración y resultado fue realizado desde diversidad de enfoques que abarcaron diversas áreas o aspectos competenciales de educación primaria. Las técnicas caminaron desde las realizadas, sobre todo bajo parámetros plásticos, cajas en las que el concepto era el valor primordial u otras, en las que los recursos audiovisuales fueron la base constructiva para la creación artística. 


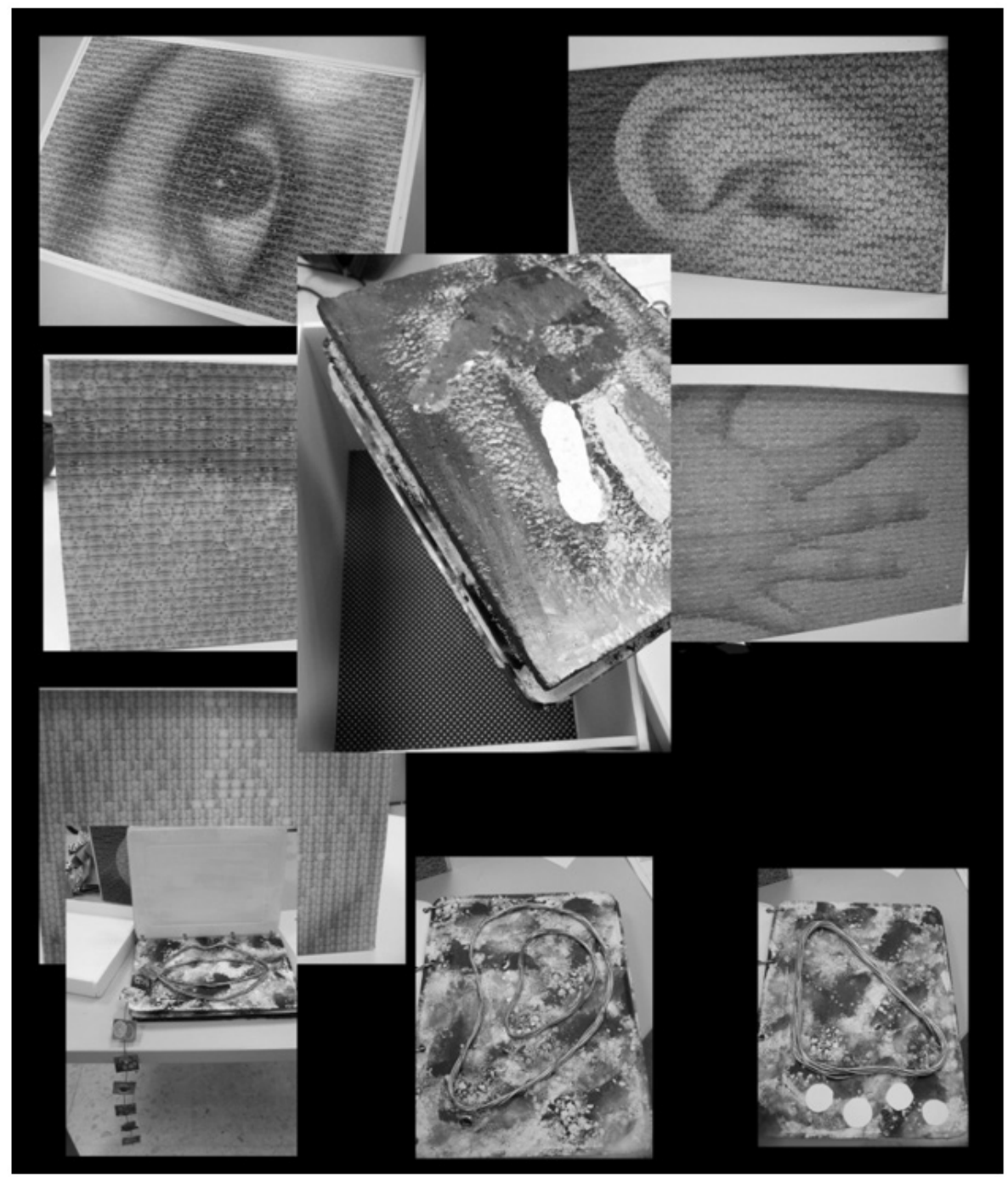

Figura 3. Caja de los cinco sentidos. Obra de Beatriz Sais Pascual. La caja trata sobre el contenido curricular "Los cinco sentidos", contemplado en el área de ciencias naturales de $1^{\circ}$ de primaria. La autora plantea cinco montajes fotográficos realizados con un programa digital. Partiendo de imágenes de su rostro, realizó fotos a sus 58 compañeros y compañeras de grupo, de modo que, mediante el proceso de discriminación de tonos del programa se configura la imagen final expuesta en cinco caras de la caja. El contenido consiste en un libro de artista artesanal de cinco páginas con imágenes, muestras de olores y elementos sonoros. 
Resultados y discusión. Apreciación de los estudiantes sobre el desarrollo del proyecto.

Una vez realizado el proyecto "Caja de artista" se llevó a cabo una recogida de datos sobre diferentes aspectos sobre la realización, dimensión académica y dimensión profesional, con preguntas sobre la posible aplicación didáctica en su labor como futuro docente. Los aspectos a valorar se realizaron en una escala del 1 al 6 , siendo el 1 considerado como como "poco" y el 6 como "mucho". En base a los cuestionarios individuales y anónimos que se pasaron a los estudiantes, el 75\% calificó la propuesta de altamente positiva y un 35\% entre positiva e interesante. Ante preguntas tales como "qué grado de desarrollo sobre los resultados de aprendizaje asociados a este proyecto de la guía docente de la asignatura has alcanzado? el 80\% consideró que había conseguido evidenciar muy satisfactoriamente los resultados de aprendizaje asociados a este proyecto tales como "realizar obras a través de códigos artísticos" o "conocer y utilizar recursos materiales, técnicos y documentales adecuados para la confección de proyectos novedosos en el ámbito docente".

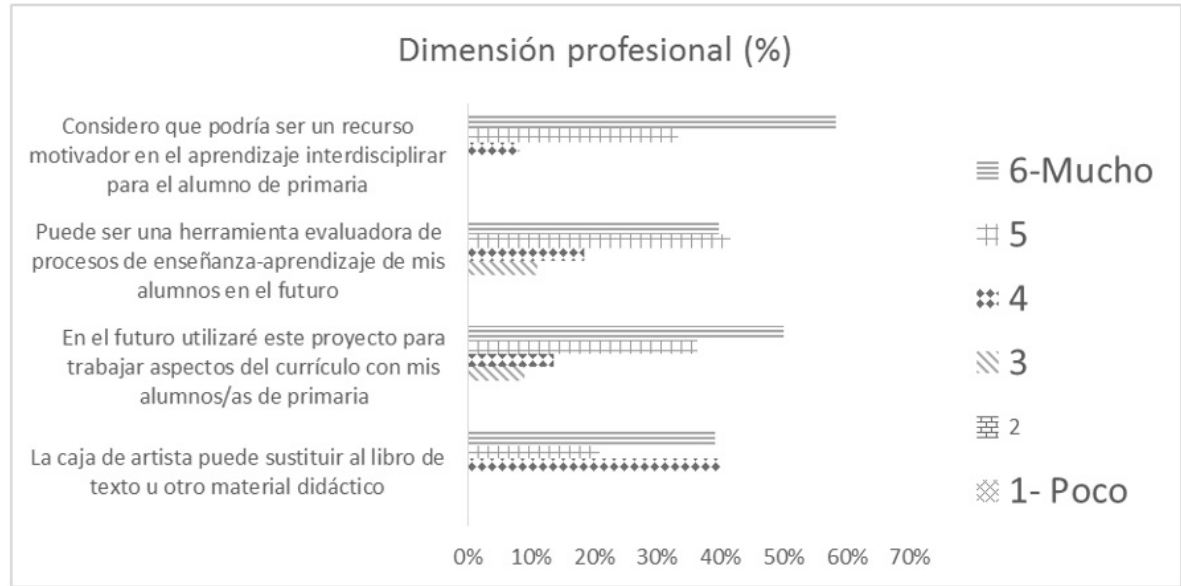

Figura 6. Valoración de los estudiantes sobre aspectos profesionales del proyecto. Fuente propia.

En base a los cuestionarios pudo extraerse que los estudiantes valoraron el sentido de utilidad de "La caja de artista", el potencial didáctico para su labor docente, la creatividad desarrollada y profusión de técnicas que habían puesto en práctica. 
Ante la pregunta abierta "en caso de que este proyecto te haya resultado enriquecedor explica el motivo", la respuesta que los estudiantes transmitieron fue que el proyecto había sido enriquecedor a nivel personal por: "su finalidad", "por ser útil para el futuro", "sus aplicaciones en el aula", "el resultado obtenido, ya que me sirve como recurso para el futuro" "mostrar una nueva forma de impartir conocimientos a mis alumnos", "lo podré utilizar en el futuro", "ser una buena herramienta de trabajo", "porque enseñas y aprendes de manera diferente y motivadora", "una herramienta útil", "nueva metodología", "en un futuro podré emplearIo", "una herramienta nueva de trabajo muy útil". Estas apreciaciones evidencian que algunos estudiantes entendieron que el proyecto podría ser un material didáctico útil para su futura labor docente. En otros casos, las respuestas aludían a su formación académica general manifestando el interés que les había supuesto el proceso debido a: "la información que ha debido ser procesada", "la cantidad de recursos utilizados", "he desarrollado mi creatividad", "ejercitar la imaginación", "los contenidos aplicados", "tener que emplear recursos teóricos y prácticos de diferentes áreas". También hubo respuestas sobre aspectos propios de la asignatura: "las distintas técnicas y materiales utilizados", "me ha permitido conocer diferentes técnicas plásticas y ha sido algo novedoso". Algunos estudiantes destacaron del proyecto:"recurso muy atractivo", "la novedad y la originalidad del proyecto", "ser innovador", "un buen proyecto", "es creativo", "un proyecto aconsejable".

Por otro lado, los alumnos consideraron el proyecto complejo o costoso debido a "el tiempo que ha sido necesario emplear en su fases, (demasiado)" (10) o "el poco tiempo estipulado en el aula para su realización". Encontraron dificultad en "entender la idea del proyecto planteado", "dificultad en plasmar lo que tenía en la mente", "conseguir unificar estéticamente todas las partes",(5) o "relacionar el contenido", "proceso de elaboración complejo", "trasladar los temas a imágenes y elementos de la plástica", "decidir el tema a tratar" (8), "ejecutar técnicas plásticas", "el propio proceso de construcción y cohesión con el contenido teórico", "elegir qué recurso para la realización, cada vez se me ocurrían más y más". Realmente la coincidencia de estas respuestas en varias ocasiones, hace pensar que realmente se trataba de un proyecto que abre muchos caminos a tratar, conceptos, técnicas plásticas y materiales. Varios manifestaron en los cuestionarios y durante el proceso de realización, la dificultad de diseñar contenidos o temas curriculares 
bajo parámetros artísticos, añadiendo a la dificultad el hecho de estar acotados en un marco conceptual-objetual como es una caja.

El tiempo, para algunos "escaso", para otros "excesivo", hace pensar que se trata de un proyecto difícil de unificar en un tiempo estipulado en un aula-taller. El trabajo de búsqueda e investigación fue para algunos estudiantes, según manifestaron, arduo. De estos y otros factores puede deducirse que cuanto menos, este proyecto no dejó indiferente a los estudiantes, y que respuestas sobre las dificultades no interfirieron en el grado de satisfacción sobre el resultado del proyecto o la respuesta sobre si desarrollarían este proyecto con sus futuros alumnos que en un $90 \%$ fue afirmativa.

Las respuestas que los estudiantes dieron a la invitación de cambio de nombre del objeto artístico del proyecto resultan de interés a la hora a atisbar nuevas posibilidades de aplicación del recurso. Los nuevos nombres elegidos para asignar el producto artístico fueron: "Caja de apoyo educativo", "La caja genio", "Caja divertida", "Caja mágica"(8), "Caja de investigación", "Caja sorpresa"(2), "El baúl de los recuerdos", "Caja de ...", "La caja de las ideas", "Caja curricular", "Historia por dentro y por fuera", "Caja creativa" (10), "Cajón de conocimiento", "Caja de contenidos", "Caja del maestro", "Imagine Box", "Caja interdisciplinar", "La caja de aprender", "Caja de aprendizaje", todos ellos hacen alusión al interés que les suscitó como posible material didáctico y recurso para trabajar, desde una óptica motivadora, contenidos curriculares en el aula de primaria.

\section{Puesta en práctica de los estudiantes en un CEIP desarrollando actividades a través de "Cajas de artista" con alumnado de educación primaria}

Una vez elaborados los proyectos, se planteó el interés a los estudiantes de comprobar la aceptación que este objeto artístico-didáctico pudiera suscitar en alumnos de primaria y profesores. Se intuyó que, como estrategia educativa, este producto realizado bajo unas premisas plásticas podía, además de ser un material evocador de procesos de enseñanzaaprendizaje, ser una motivación y un acercamiento al hecho artístico.

Se expusieron, de este modo, al equipo directivo de un CEIP, algunos ejemplos de proyectos realizados por los estudiantes, en base a los cuales éste decidió, consensuando con los docentes, qué conceptos o 
temas serían los más adecuados para los diferentes cursos de primaria. De esta manera, se eligieron doce cajas de entre las 120 realizadas por los estudiantes, y se confeccionó una actividad de dos horas que se llevó a cabo en las doce aulas ordinarias (desde $1^{\mathrm{a}}$ a $6^{\mathrm{a}}$ de primaria con dos líneas por curso) en el centro educativo. En cada aula, el maestro-tutor observó y participó de las actividades que suscitó la obra estipulada para su grupo-clase. Los estudiantes, con un compañero-acompañante, elaboraron materiales adicionales para sostener los conceptos que entrañaban las obras y, en algunos casos son los alumnos de primaria los que realizan una caja en gran grupo partiendo de las directrices del creador de la caja de artista. 

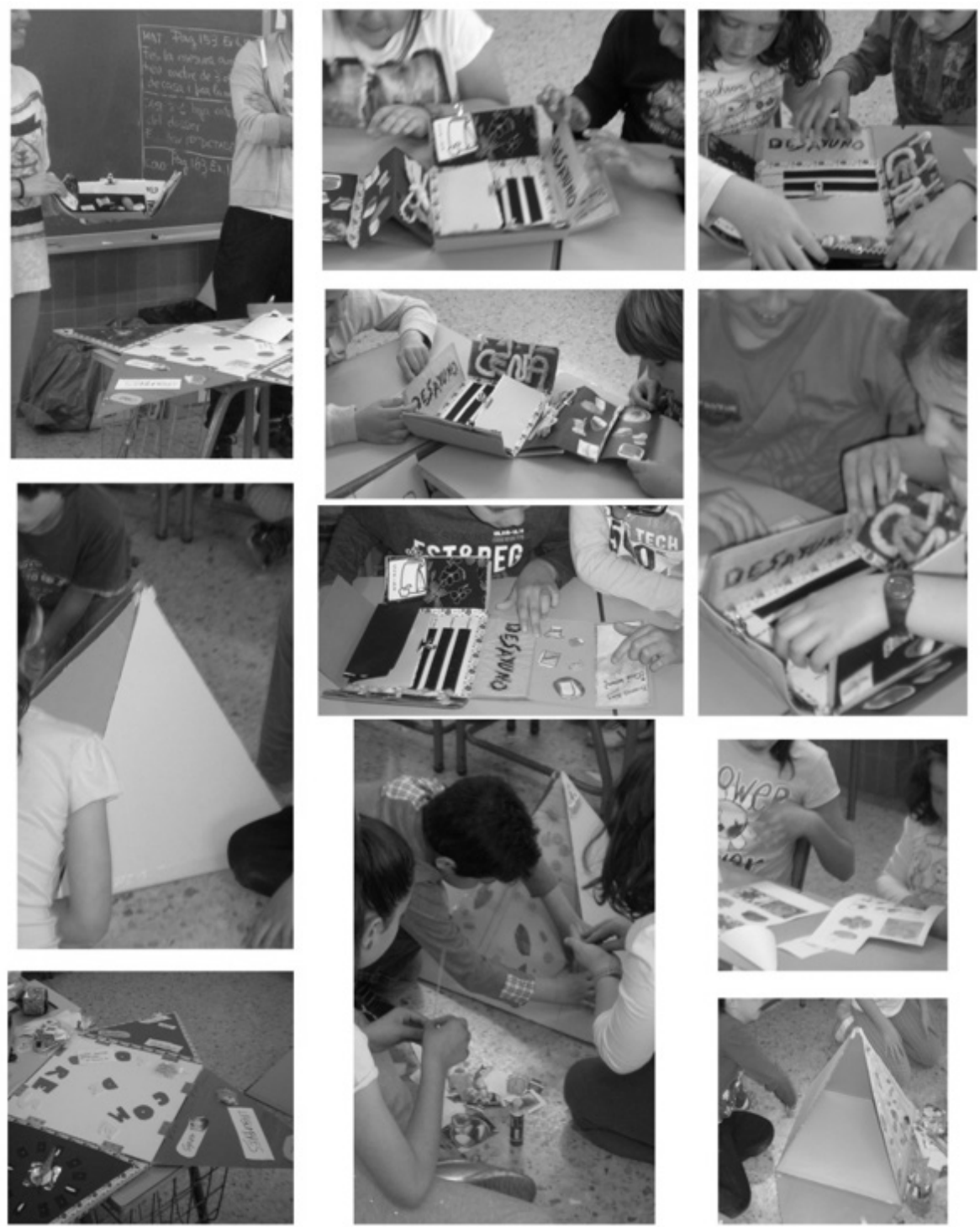

Figura 4. Narrativa sobre intervención con caja de artista “La pirámide de los alimentos" Autora Nuria Moliner Estopiñán. Los alumnos de $5^{\circ}$ de primaria confeccionan el contenido de una caja-pirámide con collages sobre los grupos de alimentos. Previamente han manipulado y debatido sobre el libro-juego. 

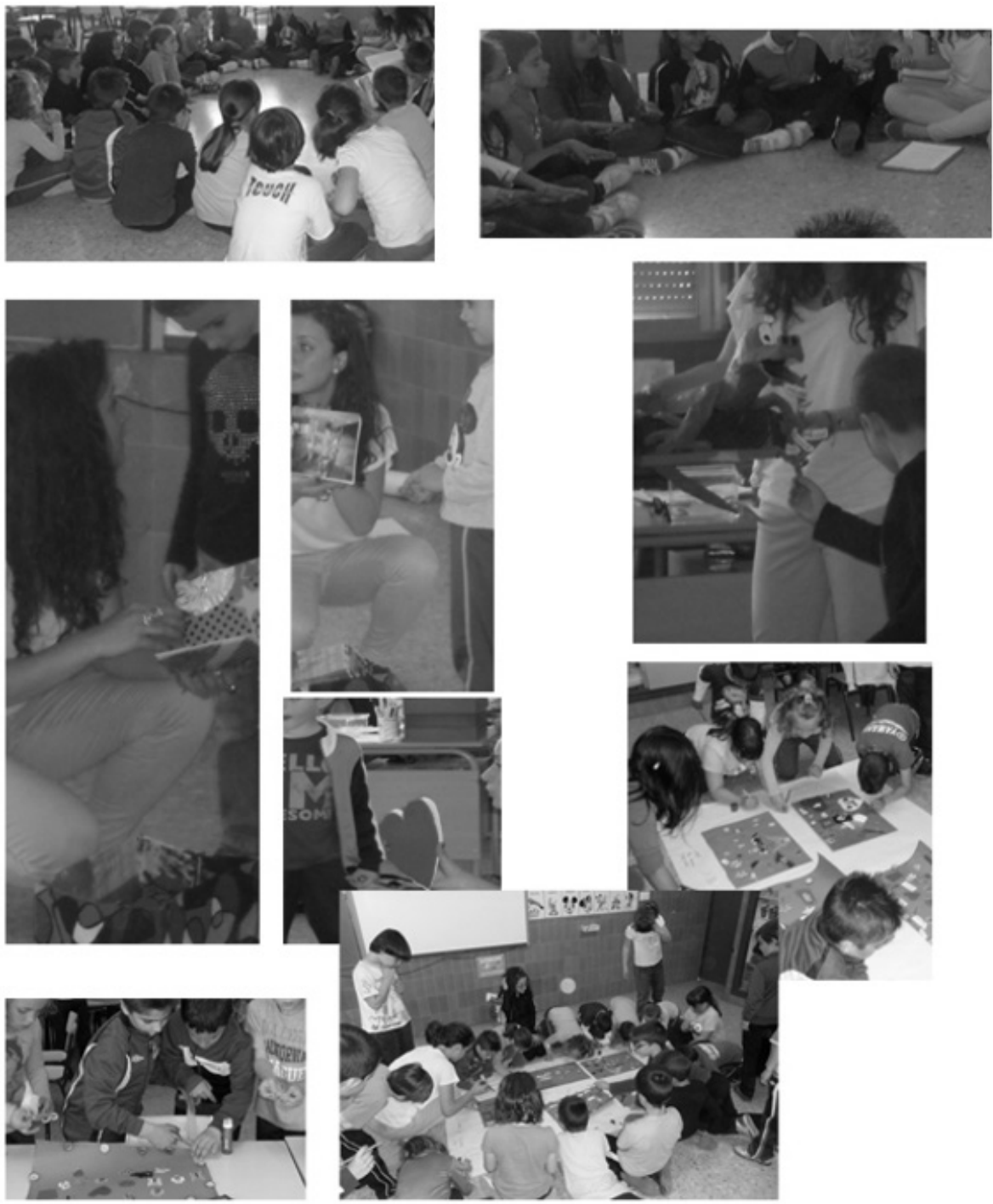

Figura 5. Narrativa sobre actividades con la caja de artista "El dragón de las emociones". Autora Paula Sanjuan García". Dicha caja alberga materiales tales como fotografías, dibujos y collages. Los alumnos de $4^{\circ}$ de primaria manipulan y participan de los conceptos sobre diferentes emociones tratadas por la autora. La caja y su contenido fueron "excusa" para incentivar la motivación de diferentes dinámicas grupales. 
Resultados y discusión. Apreciación de los docentes.

Tras la puesta en práctica, de una representación de los estudiantes en el centro educativo, los doce profesores-tutores de aula implicados, cumplimentaron un cuestionario para detectar el grado de satisfacción sobre diferentes aspectos de la puesta en práctica de los estudiantes, tales como la interacción y motivación que suscitó "La Caja de artista" en sus alumnos o, la adecuación de diseño y contenido de la caja para su curso concreto $^{3}$. Por otro lado, se realizaron preguntas sobre el interés que consideraban pudiera tener como material didáctico, -complemento o sustituto de a otros materiales más estandarizados-.

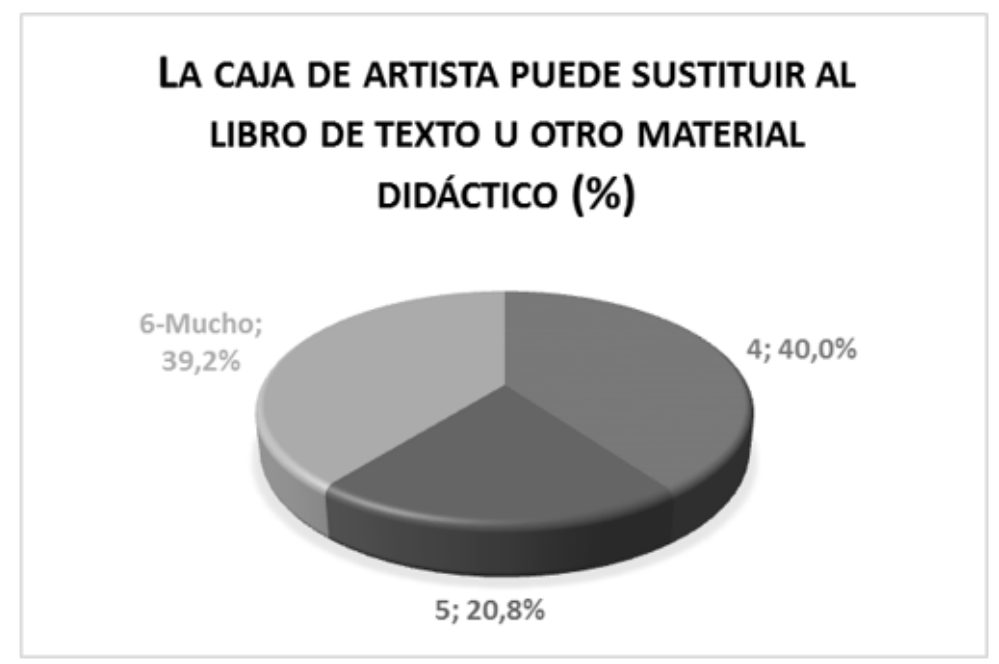

Figura 7. Valoración de los profesores sobre la posible utilización del recurso con respecto a otros materiales didácticos.

Entre sus consideraciones destacan las siguientes: el atractivo de la propuesta, destacando comentarios como: "La caja era atractiva y atrajo la atención de los niños", "La caja es muy vistosa y les impresionó a los alumnos, las fotografías y las clasificaciones son muy adecuadas para este nivel". Así mismo, consideraron que se trataba de un recurso motivador en los procesos de enseñanza-aprendizaje, algunas de sus

3 Los proyectos desarrollados en las aulas fueron: Caja de los sentidos, Pirámide de los alimentos, Caja del mar, Caja de la luz y el color, Caja de la historia, El dragón de las emociones, Caja del Pop Art vs. consumismo, Caja de las palabras, Caja de la salud, Caja de poesía, Caja de música y Caja-casa-ciudad. 
respuestas fueron: "Ios alumnos estaban muy motivados ante la presentación de un material alternativo al que están acostumbrados", "Al terminar el estudiante regaló su Caja del mar a los niños, saltaron y gritaron de alegría", "La caja era muy motivadora para los alumnos, se trabajan contenidos curriculares a partir de la plástica y el aprendizaje es más lúdico", "Resultó muy motivador porque es manipulativa y de observación". Son significativas las respuestas sobre la aplicación interdisciplinar que intuyeron los docentes: "un punto fuerte de la propuesta es conseguir representar de una manera creativa los contenidos de las áreas curriculares", "cabe destacar que es perfecto para integrar contenidos globalizados para distintas áreas".

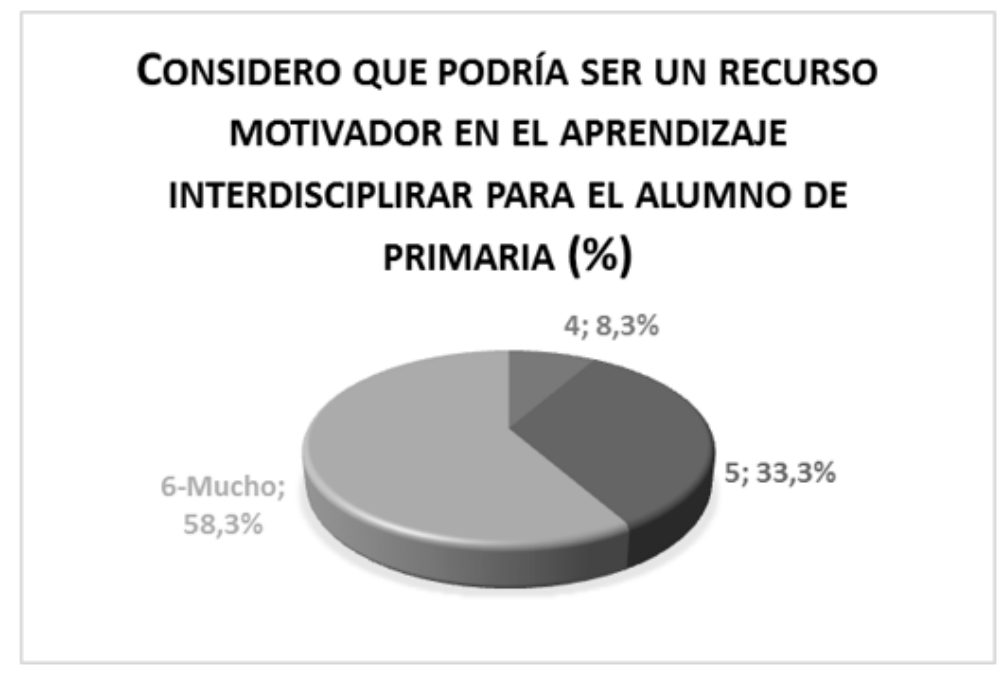

Figura 8. Valoración de los profesores sobre el carácter motivador al aprendizaje interdisciplinar del recurso.

Estos doce profesionales resumieron la razón de ser de este proyecto en unos pocos items y respuestas breves, considerando a "la caja de artista" como: "contenedor de aspectos curriculares", "proyecto interdisciplinar", "herramienta lúdica, manipulativa y de observación (añadimos, e investigación), "forma de aprender mediante las artes" o "propuesta que representa contenidos de una manera creativa y globalizadora". Cabe destacar que los docentes no conocían las bases conceptuales y didácticas del objeto artístico y, a pesar de ello, entendieron su posible utilidad en el aula. 


\section{EN EL FUTURO UTILIZARÉ ESTE PROYECTO PARA TRABAJAR ASPECTOS DEL CURRÍCULO CON MIS ALUMNOS/AS DE PRIMARIA (\%)}

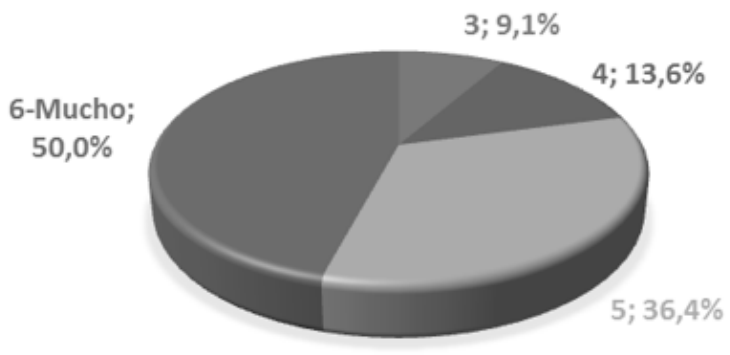

Figura 9. Valoración de los profesores sobre el interés de utilización del recurso en las aulas.

El 60\% del profesorado mostró un alto interés en realizar una Caja de artista con sus alumnos sobre un tema o unidad didáctica, los contenidos que plantearon para su posible realización en un futuro con sus alumnos oscilaron entre temas concretos como las comunidades autónomas, los seres vivos, los tipos de texto o la prehistoria hasta temas más transversales como la resolución de conflictos.

Además, se realizaron preguntas sobre las obras y las actividades relacionadas, así como sobre el interés que para ellos podía tener la colaboración de estudiantes en eventos de este tipo. Evaluaron, tanto las actividades realizadas derivadas de la Caja de artista, la propia obra artística, así como el papel docente de los estudiantes de magisterio como "altamente positiva".

\section{Conclusiones}

El proyecto "Caja de artista" ha planteado la materialización de conceptos didácticos a través de una obra artística. En este sentido, el arte conceptual brinda al estudiante la posibilidad del diálogo entre las propias ideas y el objeto artístico. La interrelación de conceptos y técnicas artísticas, ha puesto de manifiesto entendimientos y miradas personales 
diversas. De este proyecto, subyace el convencimiento de que los procesos de enseñanza-aprendizaje no pueden tratarse de un modo uniforme. Si los intereses de los discentes, hacia unas formas y técnicas artísticas son diferentes, los estilos de aprendizaje también lo son. Desde aquí se aboga por una toma de decisiones autónoma a la hora de enfrentarse a procesos creativos y a estrategias para el aprendizaje y la enseñanza.

La motivación hacia el aprendizaje, la autonomía en la toma de decisiones creativas y la utilidad de este objeto artístico, se han evidenciado en la apreciación sobre los procesos vividos por los estudiantes de magisterio. Por otro lado, como puede observarse en los resultados de la puesta en práctica con alumnado de educación primaria, así como en los resultados de las valoraciones de los profesores en activo implicados, la conceptualización de contenidos curriculares desde una perspectiva artística suscita la curiosidad hacia el aprendizaje y la participación. "La caja de artista" plantea de un modo lúdico el procesamiento y presentación de información, sirviendo además para la introducción de actividades relacionadas con los conceptos que entraña el objeto artístico.

La interconexión entre lenguajes, técnicas y áreas, es una de las demandas de la educación. En este sentido, la educación artística, con propuestas de este tipo, da respuesta a retos tales como la diversificación de recursos y materiales didácticos, así como la relación entre las distintas áreas. Por todo ello, la creación de materiales propios, tanto por parte de los docentes como de los discentes, es una motivación a la creación y, al mismo tiempo, a la adquisición de conocimientos y el desarrollo de las competencias básicas.

El proyecto "La caja de artista" ha evidenciado ser un recurso mediante el cual se procesa información, se almacenan descubrimientos y se plantean conceptos de cualquier área desde una óptica artística. El objeto conceptual propuesto, ha contribuido a desarrollar capacidades creativas, romper estereotipos, dinamizar procesos de enseñanza-aprendizaje y trabajar desde la interdisciplinaridad.

Para finalizar, teniendo en cuenta que la educación del siglo XXI no está separada del contexto artístico actual, los formadores y futuros formadores deben plantear rupturas entre las fronteras de las áreas y de las artes, confiar en los discentes como agentes activos y responsables de su aprendizaje $y$, al mismo tiempo, como creadores pertenecientes a su época y su cultura. 
Concepto y objeto. La caja de artista como recurso interdisciplinar en educación

primaria

ENCARNA MONTEAGUDO Y EVA MUÑOZ GUINEA

\section{Referencias bibliográficas}

Aguirre, I. (2005). Teorías y prácticas en educación artística, Barcelona: Octoedro.

Arnheim, R. (1993). Consideraciones sobre la educación artística, Barcelona: Paidós Ibérica.

Boch, E. (2009). Un lugar llamado escuela, Barcelona: Graò.

Duchamp, M. (1978). Escritos. Duchamp du Signe. Barcelona: Gustuvo Gili.

López, E. (2006). Poder, tradición e historia del arte: Apariencia desnuda. La obra de Marcel Duchamp. Anales de Historia del Arte, N (XVI), pp. 315-338.

Foster, H., Krauss, R., Bois, Y.A., Buchloh, B. (2006). Arte desde 1900. Madrid: Akal.

González, N. (2014). Entrevista a Vicente Rojo con motivo del Libro-Maleta de Octavio Paz/Marcel Duchamp. Les Atelierd du SAL, No 4, pp. 245-257.

MOMA (2011). Thing/thought: Fluxus Editions: 1962 to 1978. New York: Museum of Modern Art. Recuperado de https://www.moma.org/interactives/exhibitions/2011/ fluxus_editions/works/fluxus-1/index.html

UNESCO (2006) Organización de las Naciones Unidas para la Educación, la Ciencia y Cultura. Hoja de Ruta para la Educación Artística Conferencia Mundial sobre la Educación Artística: construir capacidades creativas para el siglo XXI, Lisboa, 6-9 de marzo de 2006.

Vásquez, A. (2013). Arte conceptual y posconceptual. La idea como arte: Duchamp, Beuys, Cage y Fluxus. Nómadas. Revista crítica de ciencias sociales y jurídicas, 37 (2013.1). http://dx.doi.org/10.5209/rev_NOMA. 2013.v3.n1.42567

Tonucci, F. (2005). Juegos maravillosos. En Regio Children S.r.I. (Eds.), Los cien lenguajes del niño (pp. 74-77). Barcelona: Rosa Sensat. 